\title{
IMPACTO ECONÓMICO DEL SECTOR EDUCACIÓN EN ARICA, VALDIVIA Y PUNTA ARENAS
}

\author{
MARIO GARAYa ${ }^{\text {, PATRICIO AROCA }}{ }^{\mathrm{b}} \&$ SERGIO SOZA-AMIGOc
}

\section{RESUMEN}

Este artículo analiza, desde una perspectiva económica, el impacto de los servicios de educación en Arica, Valdivia y Punta Arenas. Utilizando tablas input-output para los años 2007 y 2012, elaboradas a 25 actividades y productos, la investigación estima el aporte de la educación desde la perspectiva de los multiplicadores externos, de autoconsumo e internos de Miyazawa (1966). Luego, utilizando la técnica de Rasmussen (1956) se determinaron los tipos e importancia de las interrelaciones productivas existentes. Finalmente, usando la propuesta modificada de Soza-Amigo en 2007 para evaluar los impactos de las actividades por medio de los coeficientes de dispersión ponderados de acuerdo a las características de la economía, se responde al alcance de sus repercusiones en el sistema económico. Los resultados revelan que las actividades que poseen altos multiplicadores son claves y concentran sus efectos en torno a ellos, mientras que educación teniendo unos multiplicadores cercanos al promedio y baja interrelación con el sistema económico, exhibe impactos con mayores alcances.

PALABRAS CLAVE: desarrollo local, educación, impactos económicos, análisis input-output.

\section{ECONOMIC EFFECTS IN THE EDUCATION SECTOR AT ARICA, VALDIVIA AND PUNTA ARENAS}

\begin{abstract}
This article analyzes from an economic perspective the impact of educational services in Arica, Valdivia and Punta Arenas cities. Using input-output tables, elaborated for 25 activities and products for the period from 2007 to 2012, the research estimates the contribution of Education from the perspective of external multipliers, subsistence and internal Miyazawa (1966). Later, using the Rasmussen technique (1956) the types and importance of the existing productive relationships were established. Finally, applying the modified proposal Soza-Amigo in 2007 to assess the impacts of activities through scattering of its impact on the economic system, it is possible to estimate the repercussion on the economic system.

a Facultad de Educación y Ciencias Sociales, Universidad de Magallanes, Punta Arenas, Chile. mario.garay@umag.cl

b Escuela de Negocios, Universidad Adolfo Ibáñez, Viña del Mar, Chile. patricio.aroca@uai.cl

c Autor de Correspondencia; Escuela de Ing. Comercial, Universidad Austral de Chile, Puerto Montt, Chile. sergio.soza@uach.cl
\end{abstract}


The results reveal, that the activities that have high multipliers are key and concentrate their effects around them, while education taking a close to average and low interaction with the economic system multipliers, it exhibits greater scope impact.

KEY WORDS: local development, education, economic effects, input-output analysis.

\section{INTRODUCCIÓN}

Estudios sobre como incide la educación en las economías regionales son poco frecuentes en Chile; en general la mayoría de los estudios sobre el impacto de la educación en el país tienen una perspectiva nacional. En este contexto es de interés comprender y analizar cómo los procesos educacionales que se implementan en algunas ciudades chilenas afectan al desarrollo local.

La presente investigación quiere mostrar la importancia de la educación desde una perspectiva económica vinculada al proceso de desarrollo en las regiones extremas inmersas en un mundo globalizado. Desde esta perspectiva, la educación no está fuera de este contexto en su relación con el entorno y en su organización interna, preferentemente en las áreas de Docencia, Investigación, Desarrollo y Vinculación con el Medio, en tal línea de estudio, Soza-Amigo, trabaja con la tabla input-output de la comuna de Punta Arenas para el año 2007, desde donde obtiene sus multiplicadores de producto $e$ ingreso, concluyendo que el impacto de la Universidad de Magallanes es alto en la economía local, donde genera contratación de personal (creando empleos directos e indirectos), o demanda de insumos; a modo de ejemplo, se puede indicar que de ella surgen inversiones propias o por medios concursables, favoreciendo de ésta forma el mejoramiento de la calidad de vida de la región, lo que genera efectos positivos en la economía y por ende repercute en el desarrollo del capital humano, factor que es clave en el crecimiento de la región.

Por tal motivo, se propone que la "educación no debería considerarse como una actividad de consumo sino como una inversión que obtiene tasas muy altas de retorno así, la inversión en el mejoramiento de la calidad de la población puede aumentar significativamente las perspectivas económicas y de bienestar de las personas" (Schultz, 1981: 17). En consecuencia, la educación es fundamental para alcanzar mejores niveles de bienestar social y de crecimiento económico, lo que llevaría a disminuir las desigualdades económicas y sociales, propiciando la movilidad social de las personas al acceder a mejores empleos y tener mejor calidad de vida de la población. Es decir, la existencia de un centro de educación superior provoca un conjunto de efectos económicos, que influyen en la competitividad y crecimiento económico de la localidad en la cual está inserta.

Del mismo modo, se observa que determinar el impacto de variables como los servicios propios de la educación es importante, no sólo desde el punto de vista del valor agregado que proporciona a las personas, sino que también, desde una arista poco estudiada como lo son las repercusiones económicas que de tal actividad emanan hacia los variados sistemas económicos, aspecto que consideramos novedoso en los casos estudiados y en la forma en que se desarrolla la investigación.

En consonancia con lo planteado se revisa el impacto que tiene la Educación en los centros nodales de Arica, Valdivia y Punta Arenas, optándose por tales localidades dada la importancia económica de ellas en sus respectivas regiones. Además, la incorporación de Valdivia también obedece a que la misma, se usará como proxy o variable comparativa con el fin de tener una referencia del impacto de la educación en la actividad económica en una localidad "dedicada y dependiente de la educación" en especial, la educación superior. En tanto, Arica y Punta Arenas, se escogen debido a su ubicación geográfica que ostentan al ser zonas extremas las que, además se presentan como economías poco desarrolladas y dependientes de la producción de commodities (Soza-Amigo \& Aroca, 2011, 2013). En este sentido, el sistema público en general y, las actividades como la educación en lo particular, juegan un rol económico no evaluado en términos de las relaciones que nacen producto de la compra y venta de insumos que promuevan el desarrollo de otras actividades y a su vez, en la forma en que sus variados impactos se distribuyen 
en el sistema económico local.

El trabajo que se presenta se estructura de la siguiente manera, parte con un metodología que permite evaluar por medio de los multiplicadores de Miyazawa (1966 y 1971) los impactos externos, de retroalimentación o autoconsumo e internos de la educación; segundo, sus encadenamientos totales (directos e indirectos), indirectos totales e indirectos sobre el resto del sistema económico empleando la técnica presentada por Rasmussen (1956) y finalmente, utilizando la modificación presentada por Soza-Amigo (2007) para los coeficientes de dispersión ponderados propuestos por Rao y Harmston (1979), se cuantifica el alcance de los impactos de cada actividad, evaluando su nivel de concentración y dispersión. De los resultados se desprende que la educación en los tres centros es importante en términos de las relaciones económicas que promueven, las relaciones de intercambio en las que participan y, del alcance de sus impactos en el sistema económico donde están insertas.

\section{METODOLOGÍA}

Para determinar lo propuesto, se recurre a las tablas input-output del tipo domésticas de Arica, Valdivia y Punta Arenas datadas para los años 2007 y 2012, tablas confeccionadas a 25 ramas de actividad (ver cuadro 1 en anexo para su detalle) y se han obtenido mediante la técnica indirecta conocida como el método RAS y corregidas, según coeficientes de localización, siguiendo la propuesta de Miller y Blair (2009).

Definidas las tablas input-output que se utilizaron, la metodología de la investigación se desarrolla en tres partes, la primera es referida a los Multiplicadores de Miyazawa (1966, 1971), la segunda a los encadenamientos de Rasmussen (1956) y finalmente, se presenta el método con que se evaluará el grado de concentración o difusión de los impactos de las actividades y en especial de la educación en estos centros.

Miyazawa, a partir de la matriz inversa de Leontief (1936), propone evaluar los impactos exógenos y endógenos de las actividades descomponiendo ella en el producto de tres matrices (ver en anexo, formulaciones utilizadas). A estos efectos los llama externos, de retroalimentación o autoconsumo e internos. Los efectos externos, los cuales se cuantifican con la primera matriz $\left(\mathrm{M}_{1}\right)$, se explican por dos tipos de relación, la que se da entre las ventas $\left(A_{12}\right)$, que realiza la actividad al resto del sistema económico y sus efectos directos $e$ indirectos $\left(B_{1}\right)$, efectos que a su vez se combinan con las compras de insumos $\left(\mathrm{A}_{21}\right)$, que realiza la actividad al sistema con las repercusiones (directas $e$ indirectas) que ellas tienen sobre el resto de la economía (notar que se excluyen las propias; $A_{11}$ ), con esto, logra explicar los multiplicadores o efectos externos que se forman a partir de los intercambios productivos que nacen del impacto de la actividad que se estudia. En un esquema similar, los efectos de autoconsumo o retroalimentación (matriz $\mathrm{M}_{2}$ ), expresan los efectos que tienen sobre el resto del sistema económico los incrementos productivos que se gestan desde la propia actividad hacia la economía, esto es, evalúa la propagación de los impactos interindustriales que se dan al interior de la economía originados por el propio consumo del sector para el desarrollo del mismo. Finalmente, la matriz $\mathrm{M}_{3}$, expresará los efectos internos tanto directos como indirectos de la actividad como del resto del sistema productivo.

En resumen, los multiplicadores de Miyazawa (1966), serán tres por cada actividad, los externos (matriz $\mathrm{M}_{1}$ ), los de autoconsumo (matriz $\mathrm{M}_{2}$ ) y los internos (matriz $\mathrm{M}_{3}$ ). Mientras mayores sean los valores obtenidos, mayores serán los efectos que tendrá la actividad en el sistema productivo.

En relación a los encadenamientos (ver formulaciones en anexo), estos se definen por sus variados tipos de interacción que establecen con otros sectores, esto es, hacia atrás (BL), por sus compras y hacia delante (FL), por sus ventas y en conjunto explican los tipos de conexiones que presenta la actividad con el sistema económico, en términos de la demanda de insumos y servicios que realizan (BL), como, de la oferta que hacen al sistema (FL). Desde esta perspectiva se pueden encontrar cuatro tipologías de actividades; las claves, impulsoras, base e isla. Una actividad será clave, si demanda y oferta insumos al resto del sistema por sobre la media (o uno, si sus valores están relativizados, ver Tabla 2), impulsora, si sólo demanda por sobre la media, base, si la oferta que realiza está por sobre el promedio e isla o independiente, cuando su relación tanto de demanda como oferta con otras actividades 
Tabla 2. Tipos de actividades según sus Encadenamientos Relativizados.

\begin{tabular}{ccc}
\hline & $\mathrm{BL}<1.00$ & $\mathrm{BL}>1.00$ \\
\hline $\mathrm{FL}<1.00$ & Isla & Impulsor \\
$\mathrm{FL}>1.00$ & Base & Clave \\
\hline
\end{tabular}

Fuente: En base a los trabajos de Rasmussen (1956) y Hirschman (1958).

está bajo el promedio.

Finalmente, con los índices de dispersión ponderados (en anexo sus formulaciones), se medirá el impacto, en términos de concentración, que tiene cada actividad en el sistema económico desde dos perspectivas, la demanda y oferta de insumos y productos que se realiza hacia el resto del sistema. Un alto valor del índice será indicativo de concentración, por tanto, existirá baja dispersión de efectos o pocas repercusiones en el sistema. En otros términos, si $v_{i}^{w}$ es bajo, señalará que cuando aumenta la demanda de una actividad, también aumentará la demanda de todo el sistema económico de forma relativamente homogénea, por otra parte sí, $v_{i}^{w}$ también es bajo, indicará una dependencia uniforme de la actividad en la estructura productiva, lo contrario, se interpreta como una relación puntual con el sistema. La ventaja de este índice es que el mismo se pondera en función del grado de desarrollo que posea la economía, las variables que se utilizan son la demanda final o producto total y, además considera el modelo de Leontief (1936) para su enfoque de demanda y el de Ghosh (1968) para la oferta.

Una vez relativizados los índices, si ambos están bajo la media, indicará que los impactos de la actividad son uniformes en el sistema económico, por tanto, las modificaciones que emanen de ella serán impulsoras de dinamismo en la economía. Si es solo $v_{j}^{w}$ presenta un valor bajo, implicará que sus repercusiones son importantes desde la óptica de la demanda de insumos o productos, no afectando en términos de oferta, situación similar ocurrirá con el $v_{i}^{w}$.

\section{RESULTADOS}

Revisados los principales resultados de los multiplicadores de Miyazawa (1966) referidos al sistema en general y a los propios del servicio educación (s-23), se observó que para el año 2012 en Arica, la actividad que presentaba un mayor efecto multiplicador externo fue los servicios de transporte terrestre (s-15), de autoconsumo la industria manufacturera no metálica (s-08), e interno, minería (s-07). En el caso de los servicios de educación, el efecto externo si bien estaba bajo la media, estuvo muy próximo a la misma, su valor se justifica por lo alto del impacto del transporte terrestre que sube el promedio, sin embargo, si se excluye tal rama, se observa que los efectos multiplicadores de los servicios educacionales aparecen entre los cuatros más altos. En relación a sus efectos de autoconsumo, fueron bajos, esto es, no impactó sobre sí mismo y, los internos resultaron ser altos.

En síntesis, los servicios de educación en Arica, generan altos multiplicadores de impacto en el sistema económico, se presentan como una actividad relacionada con el resto de la economía, no siendo motivo de efectos de demanda su propia dependencia. En relación al año 2007, en general, los efectos externos y de autoconsumo fueron similares para el sistema no observándose diferencias, sin embargo, los efectos internos se manifestaron cerca del promedio, pero por debajo. En tal sentido, los efectos multiplicadores de los servicios de la educación en Arica, han favorecido el desarrollo de la localidad, en especial por medio de sus efectos externos.

Tanto en Valdivia como en Punta Arenas, para los años 2007 y 2012, se detectaron similares comportamientos y motivadores de dinámica como los de Arica. Lo interesante de ello es que en ambos años y comunas los multiplicares en general, fueron muy próximos al promedio, lo que se interpreta como efectos globales comunes e impactos tan relevantes como el resto de las actividades, en tal lógica, la educación contribuye al desarrollo económico en ellas en forma importante, en especial en Valdivia. Otro resultado curioso, para esta última localidad, es que los servicios de autoconsumo de la educación contribuyen a demandar más insumos, aspecto que en Arica era 
bajo y en Punta Arenas, cercano al promedio.

En relación a los encadenamientos, tanto para el año 2007 como 2012, en las tres comunas y comparando las tres formas de detectar sus interrelaciones, se observó que las actividades más importantes fueron; productos agrícolas (s-01), ganado; productos pecuarios y carne (s03), pescados (s-05), industrias (s-08 y s-09), en 2012 y; electricidad (s-10), comercio (s-13) y comunicaciones (s-18) en 2007. Los servicios de la educación, no afectarían el sistema en términos de demanda y oferta de insumos, en tal sentido, estas últimas técnicas direccionan la afirmación que la educación es una actividad aislada dentro del sistema económico, independientemente de la forma en que se midan sus interrelaciones (efectos totales, indirectos totales e indirectos sobre el resto del sistema económico); esto es, si bien los servicios de educación forman parte del sistema económico, sus interrinterrelaciones con el mismo son escasas $y$ poco gravitante en un sentido de requerir $y / 0$ generar insumos o productos finales desde y hacia el resto de la economía.

Un aspecto curioso, es la evolución de las relaciones, en general, en los tres centros, se hace cada vez más importante el sector industrial y las actividades propias del turismo (comercio (s-13, hotelería y restaurantes ( $s-14)$, transporte, comunicaciones y servicios relacionados (s-15 a $\mathrm{s}-18$ ), pero se pierde importancia en construcción (s-12), en tal sentido, suele existir una relación entre este último y la educación, pues a mayor población, se asume que existe un incremento de la construcción, y a mayor población y construcción, es usual la presencia de un efecto alométrico, por tanto deberían existir más requerimientos por los servicios educacionales, en esta línea, la pérdida de interrelaciones de los productos de la construcción, sumado a los multiplicadores de Miyazawa (1966) que se presentan, se puede asumir que están repercutiendo en un incremento de los servicios de educación, entendidos estos, como la relación anteriormente descrita.

Al conjugar las dos últimas técnicas y centrados en el caso de los servicios de educación, los resultados en conjunto señalan por un lado que, los efectos externos, de autoconsumo e internos, si bien están dentro del promedio, sus relaciones interindustriales no alcanzan a estar sobre éste, lo que explica los resultados obtenidos, situación que se detecta en los tres centros, sin embargo, ello no implica que no tengan importantes impactos, estos se harian presente en la medida que se haga más notorio el efecto alométrico.

Luego de revisar el alcance de los impactos, utilizando como ponderadores tanto la producción total como la demanda final, una vez relativizados los resultados se detectó que, el alcance de los impactos es alto en ambas condiciones. En lo particular, luego de ponderar por la producción total ${ }^{1}$, en las tres localidades los alcances de la educación son altos, esto es, muy dispersos, no manifestando concentración de ellos, en especial en Valdivia, luego sigue Punta Arenas y finalmente Arica. Lo novedoso de estos resultados es que resulta ser un sector muy dinamizador aun cuando, desde la perspectiva de los encadenamientos, se presenta como independiente, lo que corrobora los resultados obtenidos con el multiplicador de Miyazawa (1966). El año en que más alcance logran Arica y Valdivia es el 2012, y en Punta Arenas el 2007, impactos que además se encuentran en ambos sentidos, esto es, desde la óptica de la demanda como de la oferta de insumos y productos.

Con respecto a lo anterior, si se contrasta este último enfoque con el de los encadenamientos, se detecta que las actividades que resultaron ser claves no manifiestan altos impactos en el sistema económico, es decir, son productos o servicios que si bien, demandan y ofertan por sobre el promedio, sus alcances son limitados, no así con otras actividades como los servicios de la educación, que no demanda ni oferta por sobre el promedio, pero tienen las mayores repercusiones en los distintos sistemas económicos.

Usando la demanda final como ponderador, se observó una situación similar a la anterior, sin embargo, también se detectan pequeñas diferencias, por ejemplo, en el caso de los impactos en un sentido de demanda, tanto en Valdivia como en Punta Arenas se detectó un mejoramiento de estos, no así en Arica. En un sentido de oferta, Arica mejora, Punta Arenas se mantiene, pero en Valdivia pierde relevancia, aunque la educación no impactó significativamente, sigue siendo de las actividades con mayores repercusiones sobre el

1 En este caso, un valor bajo uno se interpreta como un alcance superior al promedio y entre más cercano a cero, mayor impacto. 
sistema económico.

Finalmente, conjugando todos los resultados, se observa que en el caso de la educación, su impacto se ve aparentemente anulado, desde la perspectiva de los encadenamientos, ello ocurre dada las magnitudes de los flujos que existen entre las actividades que resultaron ser claves, sin perjuicio de ello, el impacto de los servicios educacionales, son importantes, tanto desde la perspectiva de los multiplicadores como de la dispersión de sus efectos, pero no en el sentido de la cantidad de insumos que requiere u oferta, sino desde la perspectiva de los alcances de sus impactos, esto es, los montos que se tranzan desde ella, si bien son bajos respecto a otras actividades, sus impactos son importantes.

\section{CONCLUSIONES}

Los resultados de los efectos multiplicativos de los servicios de educación, los encadenamientos que forman y los alcances que presentan en zonas extremas, Arica por el norte de Chile, Punta Arenas en la zona austral y Valdivia que se utiliza como centro de comparación en el sur de Chile, avalan la hipótesis de que tales servicios, en general, contribuyen al desarrollo desde una perspectiva económica.

Una conclusión llamativa del trabajo desarrollado, es la importancia de ciertas interrelaciones respecto al alcance de las mismas y sus efectos multiplicadores una vez que los mismos se caracterizan en externos, de autoconsumo $e$ internos, en este sentido, se detectó que existen actividades identificadas como claves, desde la técnica de encadenamientos, pero con multiplicadores que apuntan al incremento de la producción originado por el autoconsumo o efectos internos $y$, con efectos concentrados, situación contraria a la educación, donde se caracterizó por presentar altos alcances, contribuyendo más al desarrollo que las anteriores, pero con pocas relaciones interindustriales en un sentido de demanda y oferta de insumos.

Se puede concluir que los servicios de educación en zonas extremas y en localidades que se caracterizan por ser turísticas y dependientes de esta, sus efectos son, en términos de multiplicadores próximos al promedio, no poseen interrelaciones por medio de la demanda $\mathrm{u}$ oferta de insumos o productos, pero con altos alcances independientemente del ponderador utilizado.

En tal sentido, una política de desarrollo para estas localidades, pasaría por considerar que aun cuando existen actividades que aparentemente son importantes y poseen efectos concentrados, existen otras que siendo más independientes, presentan más oportunidades de desarrollo, en tal lógica, esta investigación abre un debate sobre aspectos pocas veces considerados en regiones como las aquí estudiadas, esto es, la concentración de los efectos versus sus aportes al desarrollo local y el dinamismo económico que ellos presentan como motores del mismo. Además, insta a la elaboración de otras futuras publicaciones donde se profundice sobre el rol que juegan las universidades en la dinámica expuesta dado ello, por el alto número de personas que contratan y por la compra y venta de insumos que realizan.

La entrada en vigencia de la gratuidad es un avance hacia una sociedad equitativa y responde a la concepción de la educación como derecho social, lo que tendrá un efecto directo en la economía familiar y comunal. Por lo que se espera impactos positivos en la formación del capital humano y que beneficia no sólo a los destinatarios de la acción formativa, sino que genera efectos indirectos que constituyen un beneficio para todo el sistema social en su conjunto.

Un aspecto que se puede trabajar a futuro es cómo afectaría en la estructura de consumo la posibilidad que exista gratuidad en la educación, en tal sentido el trabajo habré un campo amplio de investigación pues, por un lado, al existir gratuidad, la misma se puede financiar vía recaudación de impuestos, lo que afectaría las conductas de consumo individual; y por otra parte, al verse las personas con más ingreso dado que ya no cancelaría en educación, se presenta una oportunidad para acceder al consumo de otros bienes o servicios que antes no les era accesible, esta situación, es interesante y dependerá del saldo neto de cada contribuyente. Además, las propias instituciones también podrían cambiar sus estructuras de consumo, dado que ahora los ingresos que perciben por entregar tal servicio, serían más constantes y a corto plazo. 
Sin perjuicio de lo comentado, en una primera instancia y con un saldo neto positivo en términos de dinero para las personas, estas diferencias contribuirán a incrementar la producción en su globalidad vía aumento del consumo doméstico, posteriormente, las estructuras se debieran estabilizarse.

\section{AGRADECIMIENTOS}

Se agradece a FONDECYT por los financiamientos entregados por medio de las investigaciones "elementos de economía regional para la política educacional en Chile", Proyecto Número 1140082 y, "La Vocación Productiva, el Nivel Educativo y la Funcionalidad del Centro Emisor y Receptor como Factores Explicativos de la Conmutación Inter e Intrarregional en la Región de los Andes Australes de la Patagonia Chilena", Proyecto Número 1160196. También se agradece el financiamiento parcial otorgado por el Centro de Estudios de Conflicto y Cohesión Social del Programa CONICYT/FONDAP/15130009 de la República de Chile y finalmente, a Fondef por medio del proyecto IDeA "medidor del impacto y derrames de proyectos regionales en Chile", número CA13i10061.

\section{BIBLIOGRAFÍA}

Aroca, P., \& Soza-Amigo, S. (2013). "Diferencias Productivas Estructurales Entre el Centro y la Periferia: Magallanes y Arica versus el Promedio Nacional". Magallania, 41 (2), 55-75.

Ghosh, A. (1968). A Note on Leontief Models with NonHomogeneous Production Functions. En Planning programming and input-output models: Selected papers on Indian planning. Monographs, University of
Cambridge Department of Applied Economics at the New York: University press.

Hirschman, A. (1958). The Strategy of Economic Development. New Haven, Connecticut, USA, Yale University Press.

Leontief, W. (1936). Quantitative Input and Output Relations in the Economic Systems of the United States. The Review of Economic Statistics, 18(3), 105-125.

Miller, R., \& Blair, P. (2009). Non Survey And Partial- Survey Methods: Fundamentals. In Input-Output: Analysis Foundations and Extensions (pp. 303-346). Cambridge: University Press.

Miyazawa, K. (1966). Internal and external matrix multipliers as a matrix. Hitotsubashi Journal of Economics, 8, $39-58$

Miyazawa, K. (1971). An analysis of the interdependence between service and goods-producing sectors, Hitotsubashi Journal of Economics, 12, 10-21.

Rasmussen, P. (1956): Studies in Inter-Sectoral Relations. Amsterdam, North- Holland P. C.

Rao, V., \& Harmston, F. (1979). Identification of Key Sectors in a Region of a Developed Economy. Annals of Regional Science, 13(3), 78-90.

Sonis, M., \& Hewings, G. (1993). Hierarchies of Regional SubStructures and their Multipliers within Input-Output Systems: Miyazawa Revisited. Hitotsubashi Journal of Economics, 34, 33-44

Soza-Amigo, S. (2007). Análisis Estructural Input-Output: Antiguos problemas y nuevas soluciones. Tesis (Doctorado en Economía Aplicada). Oviedo, España, Universidad de Oviedo, Departamento de Economía Aplicada.

Soza-Amigo, S., \& Aroca, P. (2011). Economías aisladas, pequeñas y dependientes de commodities: el caso del petróleo y metanol en la comuna de Punta Arenas, Chile. Magallania, 39(2), 113-135.

Schultz, T. (1981). Invirtiendo en la gente. La cualificación personal como motor económico. Editorial Ariel, España. 


\section{ANEXO 1}

Tabla 1. Relación entre el tipo de sector,

número de la actividad y la actividad.

\begin{tabular}{|c|c|c|}
\hline & & ACTIVIDAD \\
\hline \multirow{7}{*}{ 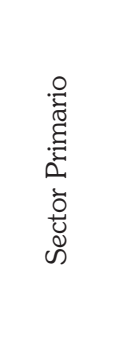 } & $\mathrm{s}-01$ & Productos agrícolas \\
\hline & $\mathrm{s}-02$ & Frutas \\
\hline & $\mathrm{s}-03$ & Ganado; productos pecuarios y carne \\
\hline & s-04 & Productos silvícolas; maderas y papel \\
\hline & s-05 & Pescados y productos del mar industrializados \\
\hline & s-06 & Petróleo crudo y combustibles y lubricantes \\
\hline & s-07 & Minería \\
\hline \multirow{2}{*}{$\begin{array}{l}\text { Sector } \\
\text { Industrial }\end{array}$} & $\mathrm{s}-08$ & Industria manufacturera no metálica \\
\hline & s-09 & Industria manufacturera metálica \\
\hline \multirow{16}{*}{ 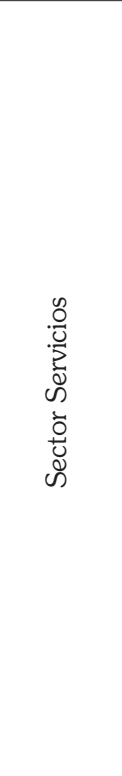 } & $\mathrm{s}-10$ & Electricidad \\
\hline & s-11 & Gas y agua \\
\hline & $\mathrm{s}-12$ & Productos de la construcción \\
\hline & s-13 & Servicios comerciales \\
\hline & s-14 & Servicios de hotelería y de restaurantes \\
\hline & s-15 & Servicios de transporte ferroviario y caminero \\
\hline & s-16 & Servicios de transporte marítimo y aéreo \\
\hline & s-17 & Servicios conexos de transporte \\
\hline & s-18 & Servicios de comunicaciones \\
\hline & s-19 & Servicios financieros y de seguros \\
\hline & $\mathrm{s}-20$ & Servicios empresariales \\
\hline & $\mathrm{s}-21$ & Servicios inmobiliarios y de propiedad de vivienda \\
\hline & s-22 & Servicios de administración pública \\
\hline & $\mathrm{s}-23$ & Servicio de educación pública y privada \\
\hline & $s-24$ & Servicio de salud pública y privada \\
\hline & $\mathrm{s}-25$ & Servicios de esparcimiento y otros servicios \\
\hline
\end{tabular}

Fórmulas utilizadas:

Multiplicadores de Miyazawa $(\mathbf{1 9 6 6}, \mathbf{1 9 7 1})$ basados en la descomposición de Sonis y Hewings (1993):

$B=\left[\begin{array}{ll}B_{11} & B_{12} \\ B_{21} & B_{22}\end{array}\right]=\left[\begin{array}{cc}\Delta_{1} B_{1} & \Delta_{1} B_{1} A_{12} B_{2} \\ \Delta_{2} B_{2} A_{21} B_{1} & \Delta_{2} B_{2}\end{array}\right]=\left[M_{1}\right]\left[M_{2}\right]\left[M_{3}\right]=\left[\begin{array}{cc}\Delta_{11} & 0 \\ 0 & \Delta_{22}\end{array}\right]\left[\begin{array}{cc}I & B_{1} A_{12} \\ B_{2} A_{21} & I\end{array}\right]\left[\begin{array}{cc}B_{1} & 0 \\ 0 & B_{2}\end{array}\right]$

Donde:

$\mathrm{a}_{\mathrm{ij}} \quad=\quad \mathrm{x}_{\mathrm{ij}} / \mathrm{X}_{\mathrm{j}}$, con $\mathrm{a}_{\mathrm{ij}} \in \mathrm{A}$.

$\mathrm{x}_{\mathrm{ij}} \quad=\quad$ Corresponde a sus inputs.

$\mathrm{X}_{\mathrm{j}} \quad=\quad$ Representa a la producción total.

$\mathrm{A}=\quad$ Matriz de Coeficientes Técnicos; $A=\left[\begin{array}{ll}A_{11} & A_{12} \\ A_{21} & A_{22}\end{array}\right]=\left[a_{i j}\right]$. 


$\begin{array}{lll}\mathrm{I} & = & \text { Matriz Unitaria; } I=\left[\begin{array}{cc}I_{11} & 0 \\ 0 & I_{22}\end{array}\right] . \\ \mathrm{B} & = & (\mathrm{I}-\mathrm{A})^{-1} ; \text { Inversa de Leontief; }\left[b_{i j}\right] . \\ \Delta_{1} & = & \left(\mathrm{I}-\mathrm{B}_{1} \mathrm{~A}_{12} \mathrm{~B}_{2} \mathrm{~A}_{21}\right)^{-1} . \\ \Delta_{2} & = & \left(\mathrm{I}-\mathrm{B}_{2} \mathrm{~A}_{21} \mathrm{~B}_{1} \mathrm{~A}_{12}\right)^{-1} . \\ \mathrm{B}_{1} & = & \left(\mathrm{I}-\mathrm{A}_{11}\right)^{-1} . \\ \mathrm{B}_{2} & = & \left(\mathrm{I}-\mathrm{A}_{22}\right)^{-1} .\end{array}$

\section{Rasmussen (1956)}

Poder de Dispersión (efectos totales)

$\mathrm{BL}_{j}^{\mathrm{R}}=\frac{\mathrm{n} i^{t}(\mathrm{I}-\mathrm{A})^{-1}}{i^{t}(\mathrm{I}-\mathrm{A})^{-1} \mathrm{i}}$

Sensibilidad de Dispersión (efectos totales;

Ghosh, 1968)

$\mathrm{FL}_{i}^{\mathrm{R}-\mathrm{G}}=\frac{\mathrm{n}(\mathrm{I}-\mathrm{D})^{-1} i^{t}}{\mathrm{i}(\mathrm{I}-\mathrm{D})^{-1} i^{t}}$

Efectos Indirectos totales (Leontief, 1936)

Efectos Indirectos totales (Ghosh, 1968)

$\mathrm{BL}_{j}^{(\mathrm{Z}-\mathrm{I})}=\frac{\mathrm{n} i^{t}[\mathrm{~B}-\mathrm{I}]}{i^{t}[\mathrm{~B}-\mathrm{I}] \mathrm{i}}$

$\mathrm{FL}_{i}^{(\mathrm{G}-\mathrm{I})}=\frac{\mathrm{n}[\mathrm{G}-\mathrm{I}] i^{t}}{\mathrm{i}[\mathrm{G}-\mathrm{I}] i^{t}}$

Efectos Indirectos sobre tras ramas (Leontief, 1936) Efectos Indirectos sobre otras ramas (Ghosh, 1968)

$\mathrm{BL}_{j}^{(\mathrm{Z}-\mathrm{z})}=\frac{\mathrm{ni}{ }^{\mathrm{t}}[\mathrm{B}-\mathrm{b}]}{i^{t}[\mathrm{~B}-\mathrm{b}] \mathrm{i}} ;$ donde $b=\left[\begin{array}{ccc}b_{11} & \cdots & 0 \\ \vdots & \ddots & \vdots \\ 0 & \cdots & b_{n n}\end{array}\right]$

$\mathrm{FL}_{i}^{(\mathrm{Z}-\mathrm{z})}=\frac{\mathrm{n}[\mathrm{G}-\mathrm{g}] i^{t}}{\mathrm{i}[\mathrm{G}-\mathrm{g}] i^{t}} ;$ donde $b=\left[\begin{array}{ccc}g_{11} & \cdots & 0 \\ \vdots & \ddots & \vdots \\ 0 & \cdots & g_{n n}\end{array}\right]$

Donde:

$\mathrm{d}_{\mathrm{ij}} \quad$ : Elemento de la Matriz de Distribución; $\mathrm{d}_{\mathrm{ij}}=\mathrm{x}_{\mathrm{ij}} / \mathrm{X}_{\mathrm{i}}$, con $\mathrm{d}_{\mathrm{ij}} \in \mathrm{D}$.

D : Matriz de distribución; $D=\left[\begin{array}{ll}D_{11} & D_{12} \\ D_{21} & D_{22}\end{array}\right]=\left[d_{i j}\right]$.

$\mathrm{G}_{\mathrm{ij}} \quad$ : Elementos de la Matriz Inversa de Ghosh. $\left[\mathrm{G}_{\mathrm{ij}}=(\mathrm{I}-\mathrm{D})^{-1}\right]=\left[\mathrm{g}_{\mathrm{ij}}\right]$.

i : Vector fila (unitario)

t $\quad$ : Indica que el vector o matriz está transpuesto.

Coeficiente de Dispersión Ponderado según Soza-Amigo (2007) basados en la propuesta de Rao y Harmston (1979):

$\mathrm{V}_{\mathrm{j}}^{\mathrm{w}} \quad$ : C. D. Ponderado; $v_{j}^{w}=\frac{\sqrt{\frac{1}{n-1} \sum_{i=1}^{n}\left(b_{i j}-\frac{x_{j}}{X} b_{. j}\right)^{2}}}{\frac{x_{j}}{X} z_{. j}}$, con $\mathrm{j}=1,2, \ldots, \mathrm{n} y, z_{\mathrm{ij}} \in \mathrm{Z}=(\mathrm{I}-\mathrm{A})^{-1}$.

$\mathrm{v}_{\mathrm{i}}^{\mathrm{w}} \quad$ : C. D. Ponderado; $v_{i}^{w}=\frac{\sqrt{\frac{1}{n-1} \sum_{j=1}^{n}\left(g_{i j}-\frac{x_{j}}{X} g_{i .}\right)^{2}}}{\frac{x_{j}}{X} g_{i}}$, con $\mathrm{i}=1,2, \ldots, \mathrm{n}, \mathrm{y} \mathrm{g}_{\mathrm{ij}} \in \mathrm{G}=(\mathrm{I}-\mathrm{D})^{-1}$.

Donde:

$\mathrm{w} \quad$ : Ponderador $=\left\{\begin{array}{r}x_{i} / X \text {, si i es un sector de una economía desarrollada. } \\ y_{i} / Y \text {, si } \text { i es un sector de una economía en vías de desarrollo. }\end{array}\right.$ 
Tabla 3. Resumen: multiplicadores de Miyazawa (1966) relativizados para el año 2012.

\begin{tabular}{|c|c|c|c|c|c|c|c|c|c|c|}
\hline \multirow{2}{*}{\multicolumn{2}{|c|}{ ACTIVIDAD }} & \multicolumn{3}{|c|}{ ARICA } & \multicolumn{3}{|c|}{ VALDIVIA } & \multicolumn{3}{|c|}{ PUNTA ARENAS } \\
\hline & & M1 & M2 & M3 & M1 & M2 & M3 & M1 & M2 & M3 \\
\hline \multirow{7}{*}{ 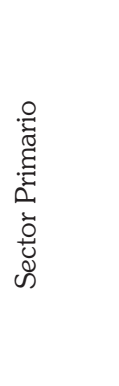 } & $\mathrm{s}-01$ & 0,90 & 0,82 & 1,04 & 0,99 & 1,00 & 1,00 & 0,98 & 0,99 & 1,02 \\
\hline & $\mathrm{s}-02$ & 0,90 & 0,79 & 1,10 & 0,98 & 0,97 & 1,03 & 0,97 & 0,96 & 1,04 \\
\hline & $\mathrm{s}-03$ & 0,91 & 0,85 & 1,02 & 1,00 & 1,01 & 0,98 & 0,99 & 0,99 & 1,00 \\
\hline & $\mathrm{s}-04$ & 0,90 & 0,79 & 1,05 & 0,98 & 0,98 & 1,02 & 0,97 & 0,96 & 1,04 \\
\hline & $\mathrm{s}-05$ & 0,90 & 0,84 & 1,03 & 0,98 & 1,00 & 1,01 & 0,97 & 0,96 & 1,21 \\
\hline & $\mathrm{s}-06$ & --- & -- & -- & --- & -- & -- & 0,97 & 0,97 & 1,04 \\
\hline & $\mathrm{s}-07$ & 0,40 & 1,73 & 1,60 & --- & --- & -- & 1,18 & 1,09 & 0,88 \\
\hline \multirow{2}{*}{$\begin{array}{l}\text { Sector } \\
\text { Industrial }\end{array}$} & $\mathrm{s}-08$ & 0,69 & 2,07 & 0,84 & 1,20 & 1,12 & 0,90 & 1,05 & 1,09 & 0,94 \\
\hline & $\mathrm{s}-09$ & 0,59 & 1,01 & 0,85 & 1,02 & 1,03 & 0,94 & 0,99 & 1,01 & 0,97 \\
\hline \multirow{16}{*}{ 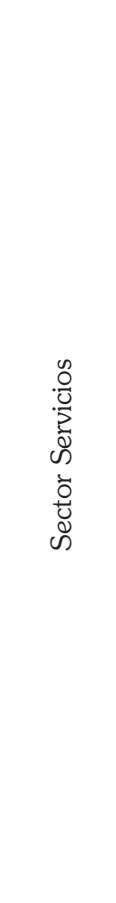 } & $\mathrm{s}-10$ & 0,88 & 0,89 & 0,95 & 0,98 & 0,99 & 1,00 & 0,98 & 0,98 & 0,99 \\
\hline & $\mathrm{s}-11$ & 0,90 & 0,80 & 1,06 & 0,98 & 0,97 & 1,04 & 0,97 & 0,98 & 1,02 \\
\hline & $\mathrm{s}-12$ & 1,01 & 0,98 & 1,00 & 1,00 & 1,02 & 0,99 & 1,05 & 1,05 & 0,94 \\
\hline & $\mathrm{s}-13$ & 0,65 & 1,54 & 0,86 & 1,10 & 1,07 & 0,88 & 1,08 & 1,05 & 0,90 \\
\hline & $\mathrm{s}-14$ & 0,91 & 0,83 & 1,03 & 0,98 & 0,99 & 1,02 & 0,98 & 0,99 & 1,01 \\
\hline & $\mathrm{s}-15$ & 5,59 & 1,15 & 1,00 & 1,01 & 1,02 & 0,95 & 0,99 & 1,01 & 0,96 \\
\hline & $\mathrm{s}-16$ & 0,94 & 0,93 & 1,01 & 0,99 & 1,02 & 0,99 & 0,99 & 1,05 & 0,99 \\
\hline & $\mathrm{s}-17$ & 0,92 & 0,86 & 1,02 & 0,98 & 0,99 & 1,01 & 0,98 & 0,99 & 1,00 \\
\hline & $\mathrm{s}-18$ & 0,87 & 0,86 & 0,95 & 0,99 & 0,99 & 1,00 & 0,98 & 0,98 & 1,00 \\
\hline & $\mathrm{s}-19$ & 0,92 & 0,85 & 1,03 & 0,98 & 0,97 & 1,04 & 0,98 & 0,98 & 1,00 \\
\hline & $\mathrm{s}-20$ & 0,02 & 1,16 & 1,19 & 1,00 & 1,01 & 0,97 & 1,00 & 1,00 & 0,95 \\
\hline & $\mathrm{s}-21$ & 0,90 & 0,81 & 1,04 & 0,98 & 0,97 & 1,03 & 0,98 & 0,97 & 1,01 \\
\hline & $\mathrm{s}-22$ & 0,64 & 1,08 & 0,93 & 0,99 & 1,01 & 1,00 & 1,02 & 1,03 & 0,98 \\
\hline & $\mathrm{s}-23$ & 0,94 & 0,89 & 1,02 & 0,99 & 1,00 & 1,00 & 0,98 & 0,98 & 1,01 \\
\hline & $\mathrm{s}-24$ & 0,89 & 0,81 & 0,98 & 0,98 & 0,99 & 1,03 & 0,98 & 0,97 & 1,05 \\
\hline & $\mathrm{s}-25$ & 0,97 & 0,90 & 1,04 & 0,98 & 0,96 & 1,05 & 0,97 & 0,95 & 1,05 \\
\hline
\end{tabular}

Donde: Efectos Externos [M1], de retroalimentación o autoconsumo [M2] e Internos [M3]. 
Tabla 4. Resumen: multiplicadores de Miyazawa (1966) relativizados para el año 2007

\begin{tabular}{|c|c|c|c|c|c|c|c|c|c|c|}
\hline \multirow{2}{*}{\multicolumn{2}{|c|}{ ACTIVIDAD }} & \multicolumn{3}{|c|}{ ARICA } & \multicolumn{3}{|c|}{ VALDIVIA } & \multicolumn{3}{|c|}{ PUNTA ARENAS } \\
\hline & & M1 & M2 & M3 & M1 & M2 & M3 & M1 & M2 & M3 \\
\hline \multirow{7}{*}{ 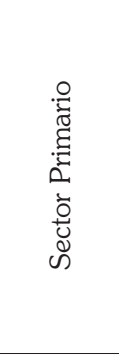 } & $\mathrm{s}-01$ & 1,01 & 0,97 & 0,90 & 0,99 & 1,00 & 0,99 & 0,97 & 0,96 & 1,01 \\
\hline & $\mathrm{s}-02$ & 1,01 & 0,96 & 0,94 & 0,99 & 0,99 & 1,01 & 0,97 & 0,98 & 1,01 \\
\hline & $\mathrm{s}-03$ & 1,01 & 0,96 & 0,90 & 1,02 & 1,01 & 0,95 & 1,03 & 1,00 & 0,96 \\
\hline & $\mathrm{s}-04$ & 1,01 & 0,96 & 0,94 & 1,00 & 1,01 & 0,99 & 0,98 & 0,96 & 1,01 \\
\hline & $\mathrm{s}-05$ & 0,99 & 0,96 & 0,67 & 0,98 & 0,98 & 1,02 & 1,02 & 1,05 & 1,68 \\
\hline & $\mathrm{s}-06$ & --- & --- & --- & --- & --- & --- & 0,98 & 0,98 & 0,95 \\
\hline & $\mathrm{s}-07$ & 1,01 & 0,99 & 4,14 & --- & --- & --- & 0,97 & 1,07 & 0,98 \\
\hline \multirow{2}{*}{$\begin{array}{c}\text { Sector } \\
\text { Industrial }\end{array}$} & $\mathrm{s}-08$ & 1,02 & 1,03 & 0,89 & 1,06 & 1,06 & 0,95 & 1,26 & 1,37 & 0,71 \\
\hline & $\mathrm{s}-09$ & 0,97 & 1,17 & 0,81 & 0,98 & 0,99 & 1,02 & 0,97 & 0,93 & 1,02 \\
\hline \multirow{16}{*}{ 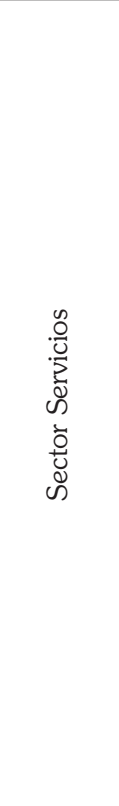 } & $\mathrm{s}-10$ & 1,01 & 0,96 & 0,90 & 0,98 & 0,96 & 1,05 & 0,97 & 0,94 & 1,00 \\
\hline & $\mathrm{s}-11$ & 1,01 & 0,96 & 0,90 & 0,98 & 0,95 & 1,06 & 0,97 & 1,00 & 1,00 \\
\hline & $\mathrm{s}-12$ & 1,02 & 1,01 & 0,89 & 1,10 & 1,09 & 0,94 & 1,05 & 1,04 & 0,95 \\
\hline & $\mathrm{s}-13$ & 0,79 & 1,13 & 0,79 & 1,01 & 1,02 & 0,96 & 1,04 & 1,01 & 0,94 \\
\hline & $\mathrm{s}-14$ & 1,01 & 1,00 & 0,89 & 0,98 & 1,00 & 1,01 & 0,98 & 1,04 & 0,99 \\
\hline & $\mathrm{s}-15$ & 1,02 & 1,04 & 0,89 & 1,05 & 1,05 & 0,92 & 1,02 & 0,99 & 0,94 \\
\hline & $\mathrm{s}-16$ & 1,01 & 0,98 & 0,78 & 1,01 & 1,04 & 0,98 & 0,99 & 1,01 & 0,96 \\
\hline & $\mathrm{s}-17$ & 1,01 & 0,99 & 0,90 & 0,99 & 0,99 & 0,98 & 0,98 & 0,97 & 0,98 \\
\hline & $\mathrm{s}-18$ & 1,01 & 1,00 & 0,90 & 1,00 & 1,00 & 1,00 & 0,98 & 0,95 & 1,01 \\
\hline & $\mathrm{s}-19$ & 1,01 & 0,98 & 0,89 & 0,99 & 0,99 & 1,00 & 0,98 & 0,94 & 0,99 \\
\hline & $\mathrm{s}-20$ & 1,03 & 1,03 & 0,89 & 1,00 & 1,01 & 0,97 & 0,99 & 0,95 & 0,95 \\
\hline & $\mathrm{s}-21$ & 1,01 & 0,96 & 0,89 & 0,98 & 0,98 & 1,01 & 0,98 & 0,96 & 0,99 \\
\hline & $\mathrm{s}-22$ & 1,03 & 1,04 & 0,88 & 0,98 & 1,01 & 1,01 & 1,01 & 0,99 & 0,98 \\
\hline & $\mathrm{s}-23$ & 1,01 & 0,98 & 0,91 & 0,99 & 1,00 & 1,00 & 0,97 & 0,96 & 1,00 \\
\hline & $\mathrm{s}-24$ & 1,01 & 1,00 & 0,90 & 0,98 & 0,99 & 1,04 & 0,98 & 0,98 & 1,02 \\
\hline & $\mathrm{s}-25$ & 1,02 & 1,00 & 0,90 & 0,98 & 0,97 & 1,03 & 0,97 & 0,95 & 0,99 \\
\hline
\end{tabular}


Tabla 5. Resumen: tipos de encadenamientos para el año 2012.

\begin{tabular}{|c|c|c|c|c|c|c|c|c|c|c|}
\hline \multirow{2}{*}{\multicolumn{2}{|c|}{ ACTIVIDAD }} & \multicolumn{3}{|c|}{ ARICA } & \multicolumn{3}{|c|}{ VALDIVIA } & \multicolumn{3}{|c|}{ PUNTA ARENAS } \\
\hline & & \multirow{2}{*}{$\begin{array}{c}\text { Total } \\
4\end{array}$} & \multirow{2}{*}{$\frac{(\mathrm{Z}-\mathrm{I})}{4}$} & \multirow{2}{*}{$\frac{(Z-z)}{4}$} & \multirow{2}{*}{$\begin{array}{c}\text { Total } \\
4\end{array}$} & \multirow{2}{*}{$\frac{(Z-I)}{4}$} & \multirow{2}{*}{$\frac{(Z-z)}{4}$} & \multirow{2}{*}{$\begin{array}{c}\text { Total } \\
4\end{array}$} & \multirow{2}{*}{$\frac{(Z-I)}{4}$} & \multirow{2}{*}{$\frac{(Z-z)}{4}$} \\
\hline \multirow{7}{*}{ 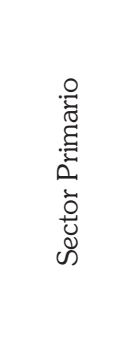 } & $\mathrm{s}-01$ & & & & & & & & & \\
\hline & $\mathrm{s}-02$ & 1 & 1 & 1 & 1 & 1 & 1 & 1 & 1 & 1 \\
\hline & $\mathrm{s}-03$ & 4 & 4 & 4 & 4 & 4 & 4 & 4 & 4 & 2 \\
\hline & $\mathrm{s}-04$ & 2 & 2 & 2 & 2 & 2 & 2 & 2 & 2 & 2 \\
\hline & $\mathrm{s}-05$ & 3 & 3 & 4 & 3 & 3 & 3 & 4 & 4 & 1 \\
\hline & $\mathrm{s}-06$ & --- & --- & --- & --- & --- & --- & 2 & 2 & 4 \\
\hline & $\mathrm{s}-07$ & 3 & 3 & 3 & --- & --- & --- & 3 & 3 & 3 \\
\hline \multirow{2}{*}{$\begin{array}{l}\text { Sector } \\
\text { Industrial }\end{array}$} & $\mathrm{s}-08$ & 3 & 3 & 3 & 3 & 3 & 3 & 3 & 3 & 3 \\
\hline & $\mathrm{s}-09$ & 4 & 4 & 4 & 4 & 4 & 4 & 4 & 4 & 4 \\
\hline \multirow{16}{*}{ 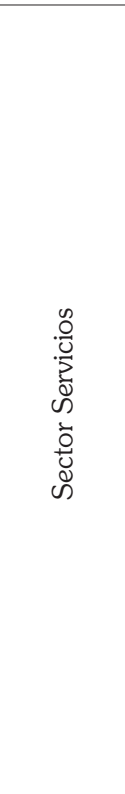 } & $\mathrm{s}-10$ & 4 & 4 & 4 & 4 & 4 & 4 & 4 & 4 & 4 \\
\hline & $\mathrm{s}-11$ & 2 & 2 & 2 & 2 & 2 & 4 & 1 & 1 & 1 \\
\hline & $\mathrm{s}-12$ & 1 & 1 & 1 & 1 & 1 & 3 & 1 & 1 & 1 \\
\hline & $\mathrm{s}-13$ & 4 & 4 & 3 & 4 & 4 & 3 & 3 & 3 & 3 \\
\hline & $\mathrm{s}-14$ & 1 & 1 & 1 & 1 & 1 & 1 & 1 & 1 & 1 \\
\hline & $\mathrm{s}-15$ & 4 & 4 & 4 & 4 & 4 & 4 & 4 & 4 & 4 \\
\hline & $\mathrm{s}-16$ & 3 & 3 & 3 & 3 & 3 & 3 & 3 & 3 & 3 \\
\hline & $\mathrm{s}-17$ & 2 & 2 & 2 & 2 & 2 & 4 & 2 & 2 & 4 \\
\hline & $\mathrm{s}-18$ & 4 & 4 & 2 & 4 & 4 & 4 & 4 & 4 & 2 \\
\hline & $\mathrm{s}-19$ & 2 & 2 & 2 & 2 & 2 & 2 & 2 & 2 & 2 \\
\hline & $\mathrm{s}-20$ & 2 & 2 & 2 & 2 & 2 & 2 & 2 & 2 & 2 \\
\hline & $\mathrm{s}-21$ & 1 & 1 & 2 & 1 & 1 & 2 & 1 & 1 & 2 \\
\hline & $\mathrm{s}-22$ & 1 & 1 & 1 & 1 & 1 & 1 & 1 & 1 & 1 \\
\hline & $\mathrm{s}-23$ & 1 & 1 & 1 & 1 & 1 & 1 & 1 & 1 & 1 \\
\hline & $\mathrm{s}-24$ & 1 & 1 & 1 & 1 & 1 & 1 & 1 & 1 & 1 \\
\hline & $\mathrm{s}-25$ & 1 & 1 & 1 & 1 & 1 & 1 & 1 & 1 & 1 \\
\hline
\end{tabular}

Donde: Clave (4), Impulsor (3), Base (2) e Isla (1).

Fuente: En base a los trabajos de Rasmussen (1956) y Hirschman (1958). 
Tabla 6. Resumen: tipos de encadenamientos para el año 2007.

\begin{tabular}{|c|c|c|c|c|c|c|c|c|c|c|}
\hline \multirow{2}{*}{\multicolumn{2}{|c|}{ ACTIVIDAD }} & \multicolumn{3}{|c|}{ ARICA } & \multicolumn{3}{|c|}{ VALDIVIA } & \multicolumn{3}{|c|}{ PUNTA ARENAS } \\
\hline & & \multirow{2}{*}{$\frac{\text { Total }}{4}$} & \multirow{2}{*}{$\frac{(Z-I)}{4}$} & \multirow{2}{*}{$\frac{(Z-z)}{4}$} & \multirow{2}{*}{$\frac{\text { Total }}{4}$} & \multirow{2}{*}{$\frac{(\mathrm{Z}-\mathrm{I})}{4}$} & \multirow{2}{*}{$\frac{(Z-z)}{4}$} & \multirow{2}{*}{$\frac{\text { Total }}{4}$} & \multirow{2}{*}{$\frac{(\mathrm{Z}-\mathrm{I})}{4}$} & \multirow{2}{*}{$\frac{(Z-z)}{4}$} \\
\hline \multirow{7}{*}{ 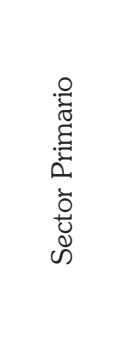 } & $\mathrm{s}-01$ & & & & & & & & & \\
\hline & $\mathrm{s}-02$ & 4 & 4 & 4 & 1 & 1 & 1 & 3 & 3 & 3 \\
\hline & s-03 & 4 & 4 & 4 & 4 & 4 & 4 & 4 & 4 & 3 \\
\hline & $\mathrm{s}-04$ & 3 & 3 & 4 & 4 & 4 & 2 & 4 & 4 & 4 \\
\hline & s-05 & 1 & 1 & 1 & 3 & 3 & 3 & 3 & 3 & 1 \\
\hline & s-06 & --- & --- & --- & -- & --- & --- & 4 & 4 & 4 \\
\hline & s-07 & 1 & 1 & 1 & -- & -- & -- & 3 & 3 & 3 \\
\hline \multirow{2}{*}{$\begin{array}{l}\text { Sector } \\
\text { Industrial }\end{array}$} & $\mathrm{s}-08$ & 3 & 3 & 4 & 3 & 3 & 3 & 4 & 4 & 1 \\
\hline & s-09 & 3 & 3 & 3 & 3 & 3 & 3 & 2 & 2 & 2 \\
\hline \multirow{16}{*}{ 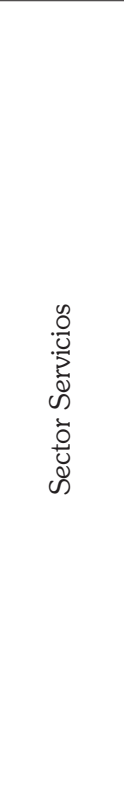 } & $\mathrm{s}-10$ & 1 & 1 & 1 & 2 & 2 & 2 & 2 & 2 & 2 \\
\hline & s-11 & 1 & 1 & 2 & 1 & 1 & 2 & 1 & 1 & 3 \\
\hline & s-12 & 1 & 1 & 1 & 3 & 3 & 3 & 3 & 3 & 3 \\
\hline & s-13 & 1 & 1 & 2 & 3 & 3 & 4 & 3 & 3 & 4 \\
\hline & s-14 & 1 & 1 & 1 & 3 & 3 & 3 & 3 & 3 & 3 \\
\hline & s-15 & 1 & 1 & 2 & 4 & 4 & 2 & 4 & 4 & 4 \\
\hline & s-16 & 1 & 1 & 1 & 3 & 3 & 3 & 4 & 4 & 4 \\
\hline & $\mathrm{s}-17$ & 2 & 2 & 2 & 4 & 4 & 2 & 4 & 4 & 4 \\
\hline & s-18 & 1 & 1 & 2 & 4 & 4 & 1 & 2 & 2 & 1 \\
\hline & s-19 & 1 & 1 & 2 & 2 & 2 & 2 & 2 & 2 & 2 \\
\hline & $\mathrm{s}-20$ & 1 & 1 & 2 & 2 & 2 & 2 & 2 & 2 & 2 \\
\hline & $s-21$ & 1 & 1 & 2 & 1 & 1 & 1 & 1 & 1 & 1 \\
\hline & $\mathrm{s}-22$ & 1 & 1 & 1 & 1 & 1 & 1 & 1 & 1 & 1 \\
\hline & $\mathrm{s}-23$ & 1 & 1 & 1 & 1 & 1 & 1 & 1 & 1 & 1 \\
\hline & $\mathrm{s}-24$ & 1 & 1 & 1 & 1 & 1 & 1 & 1 & 1 & 1 \\
\hline & $\mathrm{s}-25$ & 1 & 1 & 2 & 1 & 1 & 2 & 2 & 2 & 2 \\
\hline
\end{tabular}

Donde: Clave (4), Impulsor (3), Base (2) e Isla (1).

Fuente: En base a los trabajos de Rasmussen (1956) y Hirschman (1958). 


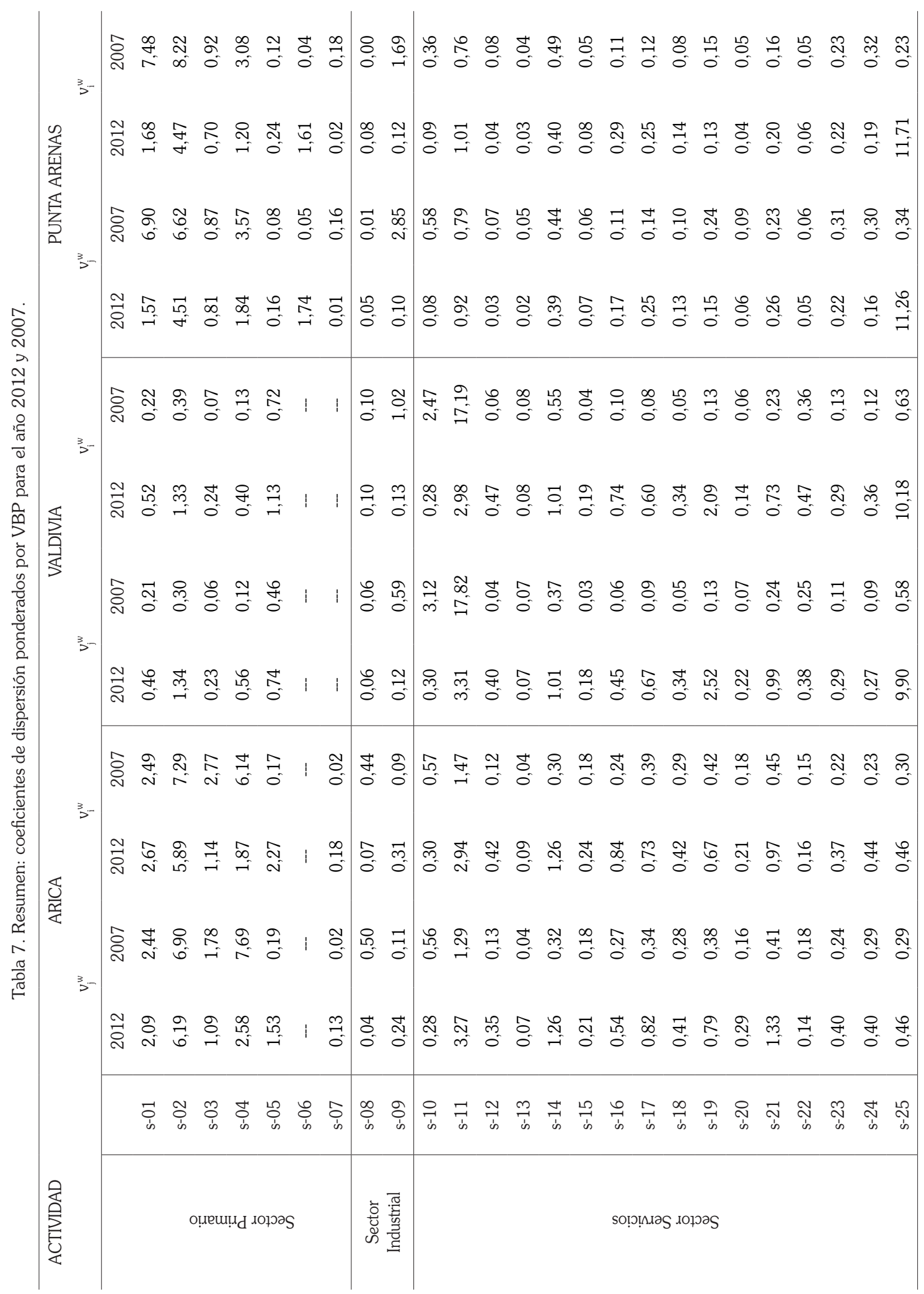




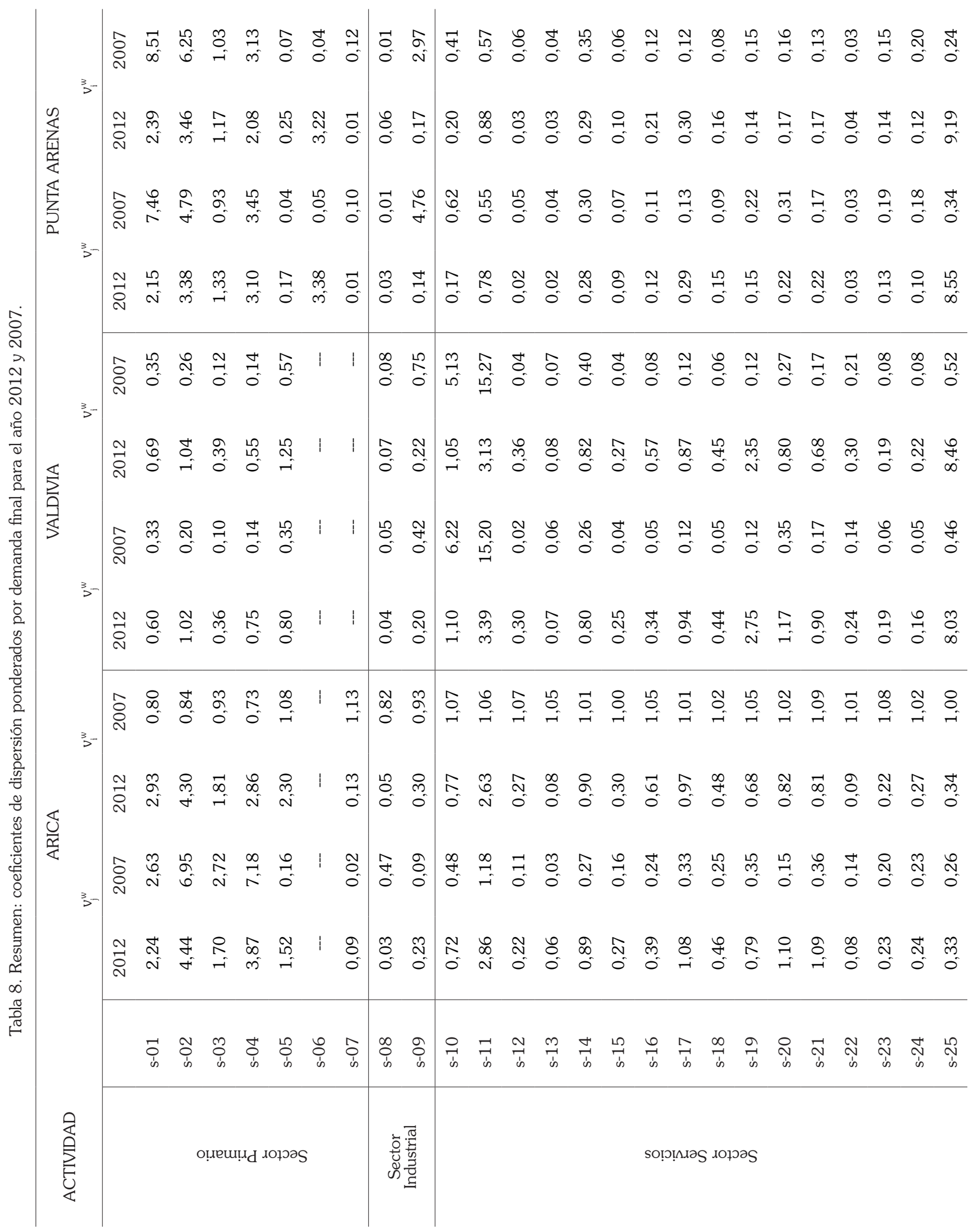


